\title{
O suicídio e sua relação com o comportamento impulsivo-agressivo
}

\author{
Gustavo Turecki ${ }^{1}$
}

\begin{abstract}
Resumo
O suicídio é um sério problema de saúde pública, principalmente em países desenvolvidos, onde as altas taxas de suicídio entre jovens adultos do sexo masculino fazem com que este seja uma das principais causas de morte e de anos potenciais de vida perdidos. A etiologia do suicídio é certamente complexa, com diversos fatores contribuindo para a predisposição a este evento. Entre estes se encontram os fatores genéticos. Nos últimos anos, diversos estudos genético-epidemiológicos têm consistentemente sugerido que o componente genético é significativo. Entretanto, o modo exato através do qual os genes aumentam a predisposição de certos indivíduos a cometer o suicídio é ainda desconhecido. Há evidência crescente de que os fatores genéticos devem influenciar a predisposição ao suicídio via uma modulação dos comportamentos impulsivo e impulsivo-agressivo. Este artigo revisa e discute os estudos que investigaram fatores genéticos no comportamento suicida, assim como esta relação com os traços impulsivo-agressivos.
\end{abstract}

\section{Descritores}

Suicídio; comportamento impulsivo; comportamento impulsivo-agressivo; genética

\begin{abstract}
Suicide is a serious public-health problem, particularly in developed countries, where high suicide rates among young males are considerably high. Indeed, suicide is one of the leading causes of death and of potential years of life lost. The etiology of suicide is certainly complex, with different factors likely playing an important role. Among these are genetic factors, as consistently suggested by several genetic-epidemiological studies that have been carried out over the last years. However, the exact mechanism by which genes increase susceptibility to suicide is not known. Several lines of evidence suggest that genes may predispose to suicide by modulating impulsive and impulsive-aggressive behaviors. In this review, studies that investigated the role of genetic factors in suicidal behavior, as well as the relationship to impulsive-aggressive traits, arediscussed.
\end{abstract}

\section{Keywords}

Suicide; impulsive behavior; impulsive-agressive behaviors; genetics

\section{Introdução}

O comportamento suicida é comumente classificado em três diferentes categorias ou domínios: ideação suicida, tentativas de suicídio e suicídio propriamente dito . Apesar de que poucos dados são disponíveis, estudos clínicos e epidemiológicos sugerem a presença de um gradiente de gravidade e também de heterogeneidade entre estas diferentes categorias ${ }^{1}$ Assim, num dos extremos teríamos a ideação suicida - ou seja, os pensamentos, idéias e desejos de estar morto - e no outro, o suicídio completo ou propriamente dito, com as tentativas de suicídio entre estes. A presença de ideação suicida e, principalmente, de uma história positiva de tentativas de suicídio têm sido vistas como tendo um importante valor preditivo na avaliação do risco para suicídio. ${ }^{2-6}$ Entretanto, uma maior quan- tidade de estudos, principalmente prospectivos, são necessários para melhor compreender a relação entre estes diferentes tipos de manifestações do comportamento suicida. Nesta revisão focalizaremos principalmente o suicídio completo.

\section{Alguns dados epidemiológicos}

A morte através de suicídio é um sério problema de saúde pública. Na maioria dos países, o suicídio se encontra entre as primeiras 10 causas de morte para indivíduos de todas as idades e entre as 3 primeiras causas de morte para indivíduos mais jovens (entre 15 e 34 anos). ${ }^{7}$ O suicídio é um fenômeno complexo que é provavelmente determinado pela interação de diversos fatores, entre os quais a constituição biológica do indivíduo, sua história pessoal, eventos circunstanciais, bem como

1. Professor Assistente - Departamento de Psiquiatria, Douglas Hospital Research Institute, McGill University - Montreal, Canadá 
o meio ambiente. ${ }^{8,9}$ A relação entre o transtorno mental e o suicídio já foi bem investigada. Diversos estudos utilizando a técnica de reconstrução diagnóstica conhecida como autópsia psicológica têm indicado que o transtorno psiquiátrico é um dos maiores fatores de risco para o suicídio. ${ }^{10,11}$ Enquanto o comportamento suicida é bastante freqüente entre a maioria dos grupos diagnósticos psiquiátricos, os transtornos mais prevalentes entre vítimas de suicídio são os transtorno depressivo maior e a dependência ou abuso ao álcool e/ou a outras substâncias psicoativas. ${ }^{12}$

Várias linhas de evidência sugerem que o denominador comum entre a maioria dos sujeitos que cometem suicídio é a presença de comportamentos impulsivos e impulsivo-agressivos. ${ }^{1,9}$ De fato, mais do que a metade dos casos que cometem suicídio preenchem critérios diagnósticos para transtornos da personalidade do grupo B, principalmente transtorno da personalidade limite e anti-social, dois quadros caracterizados pela importante presença de traços impulsivos e agressivos. ${ }^{12}$ Isto é consistente com uma série de estudos que vêm indicando haver, em várias categorias diagnósticas, uma correlação positiva entre a presença de traços impulsivos ou impulsivo-agressivos e a tendência a tentar manifestar o comportamento suicida. ${ }^{13-17}$

\section{Aspectos neurobiológicos do suicídio}

No decorrer dos últimos 20 anos, vários estudos neurobiológicos vêm consistentemente indicando que uma redução na atividade serotoninérgica é associada com o comportamento suicida, particularmente entre casos que apresentam altos níveis de traços impulsivos e impulsivo-agressivos. ${ }^{8}$ Estudos mais recentes têm encontrado um aumento no córtex préfrontal do binding de agonistas serotoninérgicos a receptores pós-sinápticos de serotonina, principalmente o receptor $2 \mathrm{~A} .{ }^{18,19}$ Isto sugere uma up regulation compensatória em resposta a uma atividade serotoninérgica reduzida nesta região cerebral, o que é de grande interesse já que o córtex pré-frontal esta envolvido na execução da função inibidora. De fato, lesões nesta área resultam em desinibição do impulso e do comportamento agressivo. ${ }^{8}$ Portanto, é possível que sujeitos com uma redução serotoninérgica nesta região cerebral possam ter uma maior predisposição a atuar impulsivamente e auto-agressivamente quando expostos a situações estressantes, tais como aquelas causadas pela presença de quadros psiquiátricos e, em algumas instâncias, isto poderia resultar na manifestação de comportamento suicida.

A importância do meio ambiente na etiologia do suicídio é bastante clara e intuitiva. Certo insight sobre a relação entre eventos de vida, fatores biológicos e comportamento suicida provém dos estudos conduzidos em primatas não-humanos. ${ }^{20,21}$ Macacos, quando privados do contato materno logo ao nascimento, manifestam comportamentos automutilatórios que podem servir como modelo do comportamento suicida relacionado a traços impulsivos e impulsivo-agressivos. A presença de comportamentos automutilatórios em macacos isolados é correlacionado com alterações neurobiológicas no sistema serotoninérgico semelhantes àquelas encontradas em sujeitos que cometem suicídio. ${ }^{20}$ Entretanto, não todos os macacos pri- vados de contato materno manifestam comportamento automutilatório. Além disso, a intensidade destes comportamentos é variável. Porém, é interessante notar que uma porção significativa desta variação, assim como da variação no turnover de serotonina dos animais que apresentam comportamentos automutilatórios, é controlada por fatores genéticos. ${ }^{22}$

\section{Genes e suicídio}

Isto é consistente com os dados de uma série de estudos genético-epidemiológicos que revisaremos brevemente neste artigo. Estes têm consistentemente indicado que os fatores genéticos conferem uma maior predisposição ao comportamento suicida. Estudos familiares têm apontado para a presença de agregação familiar do comportamento suicida ${ }^{23-27}$ (veja tabela 1). Alguns desses estudos conseguiram demonstrar que a transmissão do comportamento suicida é independente da segregação familiar de morbidade psiquiátrica. Por exemplo, o estudo de Egeland et al. ${ }^{27}$ na população Amish - uma sociedade bastante religiosa, socialmente coesa e com baixas taxas de abuso de álcool e drogas - é, apesar de simples, bastante ilustrativo. Estes autores claramente demonstraram que o transtorno do humor agregava em uma série de famílias nessa comunidade, mas a co-segregação com o suicídio foi somente observada em algumas destas famílias, sugerindo que: a) a presença do transtorno do humor é necessária, mas não suficiente, para a ocorrência de suicídio e b) os fatores que predispõem ao suicídio também tendem a agregar em famílias. Conclusões semelhantes foram também obtidas em outros trabalhos, inclusive o metodologicamente rigoroso estudo familiar realizado por Brent et al. ${ }^{23}$ Estes autores avaliaram as famílias de adolescentes que se suicidaram e de controles normais, investigando nos parentes de primeiro e segundo grau, através de entrevistas diretas, a presença de história de suicídio completo, tentativas de suicídio, ideação suicida, diagnósticos do eixo I e II, assim como a prevalência de traços de impulsividade e agressividade. Os resultados obtidos claramente sugeriram que o comportamento suicida, principalmente as tentativas e o suicídio propriamente dito, agrega em famílias e que esta agregação é independente da psicopatologia (tanto do eixo I como do eixo II) presente na família. Além disso, os dados deste estudo também indicaram haver cotransmissão do comportamento suicida e o comportamento agressivo, confirmando ao nível familiar aquilo sugerido por diversos estudos observacionais em indivíduos não relacionados.

Estudos de gêmeos e de adoção foram realizados na tentativa de separar o componente genético do ambiental. De modo geral, tanto os estudos de gêmeos como os de adoção encontraram evidência favorável à participação do componente genético na etiologia do suicídio, com taxas de concordância entre gêmeos monozigóticos (MZ) superiores às encontrados para gêmeos dizigóticos (DZ), assim como taxas superiores de concordância para parentes biológicos em comparação aos adotivos. Entretanto, estas taxas não são altas. Os estudos em gêmeos encontraram valores em torno de $12 \%$ para gêmeos MZe de $2 \%$ para os DZ. ${ }^{28}$ Os de adoção observaram taxas de concordância semelhantes, variando ao redor de $21 \%$ para os parentes biológicos e $0 \%$ para os adotivos. ${ }^{29}$ Os valores relativamente baixos de concordância provavelmente refletem o fato de que o suicídio é um quadro complexo, o qual deve resultar da intera- 
Tabela 1. Relação dos principais estudos familiares realizados em probandos apresentando comportamento suicida. $\lambda$ representa o risco relativo em parentes de primeiro grau.

\begin{tabular}{|c|c|c|c|c|c|c|c|c|}
\hline Estudo & Ano & Probando & $\mathrm{N}$ & $\begin{array}{l}\text { Fenótipo nos } \\
\text { parentes }\end{array}$ & Controles & $\mathrm{N}$ & $\lambda$ & Obs. \\
\hline \multirow[t]{3}{*}{ Tsuang et al. } & 1983 & Suicídio & 29 & Suicídio & $\begin{array}{l}\text { Pacientes s/ } \\
\text { suicídio }\end{array}$ & 481 & 3.8 & $\begin{array}{l}40 \text { anos de } \\
\text { seguimento }\end{array}$ \\
\hline & & & & Suicídio & $\begin{array}{l}\text { Pacientes } \\
\text { cirúrgicos }\end{array}$ & 153 & 26.3 & \\
\hline & & $\begin{array}{l}\text { Pacientes } \\
\text { psiquiátricos }\end{array}$ & 529 & Suicídio & $\begin{array}{l}\text { Pacientes } \\
\text { cirúrgicos }\end{array}$ & 165 & 7.7 & \\
\hline $\begin{array}{l}\text { Egeland } \\
\& \text { Sussex }\end{array}$ & 1985 & $\begin{array}{l}\text { Transtorno do } \\
\text { humor }\end{array}$ & 26 & Suicídio & & & & $\begin{array}{l}\text { Estudo na } \\
\text { população Amish } \\
\text { Agregaçãofamiliar } \\
\text { em famílias com } \\
\text { alta densidade de } \\
\text { afetados }\end{array}$ \\
\hline $\begin{array}{l}\text { Murphy } \\
\text { \& Wetzel }\end{array}$ & 1982 & $\begin{array}{l}\text { Tentativas de } \\
\text { suicídio }\end{array}$ & 127 & $\begin{array}{l}\text { Comportamento } \\
\text { suicida }\end{array}$ & & & & $\begin{array}{l}14 \% \text { dos casos } \\
\text { com HF+ }\end{array}$ \\
\hline Brent et al. & 1996 & Suicídio & 58 & $\begin{array}{l}\text { Suicídio } \\
\text { completo ou } \\
\text { tentativas } \\
\text { Ideação suicida }\end{array}$ & Normal & 55 & 5.3 & $\begin{array}{l}\text { Probandos } \\
\text { adolescentes }\end{array}$ \\
\hline Johnson et al. & 1998 & $\begin{array}{l}\text { Tentativas de } \\
\text { suicídio }\end{array}$ & 62 & $\begin{array}{l}\text { Suicídio completo ou } \\
\text { tentativas }\end{array}$ & Normal & 70 & 2.3 & idem \\
\hline
\end{tabular}

ção de diversos fatores, entre os quais o ambiente não compartilhado (ou seja, aquele que é próprio a cada gêmeo/ indivíduo) e que, portanto, deve manifestar-se naqueles casos mais graves e penetrantes. Assim, as taxas de concordância seriam certamente mais altas se a presença de uma série de comportamentos relacionados, tais como tentativas e comportamento agressivo, fossem considerados no outro gêmeo ou parente biológico. De fato, os dados de um estudo recente e metodologicamente rigoroso realizado em gêmeos da população geral australiana são consistentes com esta hipótese. ${ }^{30}$ Estes autores observaram que os fatores genéticos são responsáveis por aproximadamente $45 \%$ da variância fenotípica observada quando a ideação suicida e as tentativas de suicídio são considerados. De modo semelhante, Roy et al., ${ }^{31}$ estudando os co-gêmeos sobreviventes de pares DZ e MZ onde um dos gêmeos cometeu suicídio, encontraram que $38 \%$ dos gêmeos MZ sobreviventes fizeram tentativas de suicídio, enquanto nenhum dos gêmeos $\mathrm{DZ}$ sobreviventes demonstraram tal comportamento.

Além dos estudos que indicam a participação do componente genético na etiologia do suicídio, poucos estudos foram realizados na tentativa de melhor caracterizar este fator etiológico. Papadimitriou et al. ${ }^{32}$ investigaram o modo de herança do comportamento suicida em pacientes deprimidos com história de tentativa de suicídio e encontraram resultados compatíveis com herança poligênica. Entretanto, este estudo utilizou informações diagnósticas que foram obtidas indiretamente e, portanto, deve ser interpretado com cautela. Holland e Gosden ${ }^{33}$ relataram uma família onde uma translocação balanceada entre o cromossomo 6 e 11 [t(6,11)(q14.2;q25)] co-segregava com suicídio em uma família com um "sério transtorno psicótico" (segundo terminologia empregada pelos autores).

\section{Genética molecular do comportamento suicida}

Finalmente, alguns estudos moleculares têm sido realizados, principalmente nos últimos 5 anos (tabela 2). A maioria destes estudos focalizaram o gene que codifica a enzima triptofano hidroxilase (TPH) - que é a enzima cuja atividade controla a taxa de síntese da serotonina. Este interesse seguiu-se em grande parte ao relato de Nielsen et al..$^{34}$ de uma associação positiva entre um polimorfismo deste gene e a presença de comportamento suicida (história de tentativa de suicídio) entre indivíduos alcoolistas com problemas criminais. Apesar de que outros estudos encontraram dados consistentes com este primeiro relato, ${ }^{35-37}$ uma certa controvérsia existe a respeito da validade desta associação, já que alguns relatos foram negativos. ${ }^{38,39}$ Entretanto, é interessante observar que a maioria dos resultados consistentes com a presença de associação provém de estudos que investigaram pacientes com comportamento suicida que também apresentavam história de comportamento impulsivo e impulsivo-agressivo. ${ }^{34-37}$ Isto é congruente com a hipótese de que os fatores genéticos devem provavelmente modular a predisposição ao suicídio e talvez, a outros comportamentos suicidas, através de um aumento na manifestação de comportamentos do tipo impulsivo e impulsivo-agressivo. Dois estudos recentes que focalizaram traços agressivos encontraram resultados compatíveis com esta hipótese. ${ }^{40,41}$

De modo semelhante, os estudos realizados por nosso grupo também apontam no mesmo sentido. O nosso laboratório, em colaboração com o Bureau du Coroner e a Morgue de Montréal, tem estudado jovens indivíduos do sexo masculino que completaram o suicídio. Estes indivíduos são avaliados dentro de 24 horas após a morte, o material biológico (cérebro e amostras sangüíneas) é coletado e assim estes casos são incluídos em uma série de estudos neuroquímicos, genéticos e psicossociais. 
Tabela 2 - Relação dos estudos de associação com marcadores do gene ${ }^{7} \mathrm{TPH}$ (triptofano hidroxilase) realizados em sujeitos com comportamento suicida ou traços relacionados (agressividade)

\begin{tabular}{|c|c|c|c|c|c|c|c|}
\hline Estudo & Ano & Probandos & $\mathbf{N}$ & Controles & $\mathbf{N}$ & $\begin{array}{l}\text { Polimorfismo } \\
\text { TPH }\end{array}$ & Resultados \\
\hline Nielsen et al. & 1994 & $\begin{array}{l}\text { ACVAC }^{1} \text { com } \\
\text { tentativas de suicídio }\end{array}$ & 36 & $\begin{array}{l}\text { ACVAC sem } \\
\text { tentativas de suicídio }\end{array}$ & 34 & A779C & $P=0.016$ \\
\hline Abbar et al. & 1995 & Tentativas de suicídio & 62 & Normais & 52 & Ava II & NS \\
\hline Mann et al. & 1997 & $\begin{array}{l}\text { TDM }^{2} \text { com tentativas } \\
\text { de suicídio }\end{array}$ & 29 & $\begin{array}{l}\text { TDM sem tentativas } \\
\text { de suicídio }\end{array}$ & 22 & A779C & $P<0.009$ \\
\hline Bellivier et al. & 1998 & $\begin{array}{l}\mathrm{TB}^{3} \text { com tentativas } \\
\text { de suicídio }\end{array}$ & 52 & $\begin{array}{l}\text { TB sem tentativa } \\
\text { de suicídio }\end{array}$ & 87 & A218C & NS \\
\hline \multirow[t]{2}{*}{ Nielsen et al. } & 1998 & $\begin{array}{l}\text { ACVAC com } \\
\text { tentativas de suicídio }\end{array}$ & 146 & $\begin{array}{l}\text { ACVAC sem } \\
\text { tentativa de suicídio }\end{array}$ & 109 & A779C & $P=0.01$ \\
\hline & & $\begin{array}{l}\text { ACVAC com } \\
\text { comportamento } \\
\text { impulsivo e tentativas } \\
\text { de suicídio }\end{array}$ & 108 & $\begin{array}{l}\text { ACVAC com } \\
\text { comportamento } \\
\text { impulsivo e sem } \\
\text { tentativas de suicídio }\end{array}$ & 60 & & $P<0.001$ \\
\hline Furlong et al. & 1998 & $\begin{array}{l}\text { TDM e TB com } \\
\text { tentativas de suicídio }\end{array}$ & 125 & $\begin{array}{l}\text { TDM e TB sem } \\
\text { tentativas de suicídio }\end{array}$ & 114 & A218C & NS \\
\hline Kunugi et al. & 1999 & $\begin{array}{l}\text { TDM e TB com } \\
\text { tentativas de suicídio }\end{array}$ & 46 & Normais & 208 & $\begin{array}{l}\text { A218C } \\
\text { A779C }\end{array}$ & NS \\
\hline New et al. ${ }^{*}$ & 1998 & $\begin{array}{l}\text { Altos níveis de } \mathrm{ClA}^{4} \\
\text { em indivíduos com } \mathrm{TP}^{5}\end{array}$ & 21 & $\begin{array}{l}\text { Baixos níveis de } \\
\text { CIA em TP }\end{array}$ & 21 & A218C & $P<0.05$ \\
\hline Manuck et al.. & 1999 & Altos níveis de $\mathrm{CA}^{6}$ & 251 & Baixos níveis de $\mathrm{CA}$ & 251 & $\mathrm{~A} 218 \mathrm{C}$ & $P<0.03$ \\
\hline Tzenova et al. & 1999 & Suicídio completo & 56 & Normais & 108 & A218C & $P=0.0025$ \\
\hline
\end{tabular}

${ }^{1}$ Alcoolistas com comportamento violento e antecedentes criminais que apresentam história de tentativa de suicídio; ${ }^{2}$ TDM Transtorno depressivo maior; ${ }^{3}$ TB- Transtorno bipolar; ${ }^{4} \mathrm{CIA}$ comportamento impulsivo agressivo conforme as subescalas de irritabilidade e agressão da escala de hostilidade de Buss-Durkee; ${ }^{5}$ Transtornos da personalidade; ${ }^{6}$ Comportamento agressivo conforme a escala para história de vida de agressividade de Brown-Goodwin. NS - Não significativo. *Trabalhos que fizeram o grupamento dos sujeitos estudados baseado no genótipo (e não no fenótipo) e posteriormente avaliaram as taxas de comportamento impulsivo (avaliação fenotípica dimensional).

Após um período médio de 4 meses, as famílias destes sujeitos são submetidas a uma autópsia psicológica, o que permite uma avaliação diagnóstica bastante válida. ${ }^{42}$ Nós também observamos uma associação com TPH nesta amostra. Isto é particularmente interessante, já que os nossos dados sugerem que a associação é positiva para toda a amostra, mas o tamanho do efeito (effect size) é significativamente maior quando apenas indivíduos que cometeram suicídio usando métodos ditos violentos são considerados. ${ }^{37}$ Além disso, nossos estudos focalizando sobre outros genes que codificam para componentes do sistema serotoninérgico, particularmente o receptor de serotonina 2A (5HT2A), concomitantemente com resultados de estudos de 'binding' com este mesmo receptor no córtex pré-frontal destes casos que completaram suicídio, sugerem que fatores genéticos modulam o número de receptores $5 \mathrm{HT} 2 \mathrm{~A} .{ }^{43} \operatorname{Logo}$, é possível que fatores genéticos atuem modulando a variabilidade ao nível do sistema serotoninérgico, o qual, por outro lado, poderia mediar a presença de traços impulsivos e impulsivoagressivos e a predisposição ao suicídio.

\section{Conclusão}

Em conclusão, a participação do componente genético na etiologia do comportamento suicida parece ser considerável. Contudo, parece também ser claro que esta carga genética não é específica ao suicídio propriamente dito, muito menos à psicopatologia subjacente freqüentemente vista nestes casos. De fato, diversas linhas de evidência sugerem que uma série de comportamentos relacionados ao suicídio deva ser considerada de modo a melhor caracterizar-se tanto os próprios fatores genéticos, como outros fatores cujo papel é importante na predisposição a este evento tão trágico.

\section{Referências bibliográficas}

1. Mann JJ, Waternaux C, Haas GL, Malone KM. Toward a clinical model of suicidal behavior in psychiatric patients. Am J Psychiatry 1999; 156:181-9.

2. Fawcett J, Scheftner WA, Fogg L, Clark DC, Young MA, Hedeker $\mathrm{D}$, et al. Time-related predictors of suicide in major affective disorder. Am J Psychiatry 1990;147:1189-94.
3. Beck AT, Brown G, Berchick RJ, Stewart BL, Steer RA Relationship between hopelessness and ultimate suicide: a replication with psychiatric outpatients. Am J Psychiatry 1990; $147: 190-5$

4. Beck AT, Steer RA, Kovacs M, Garrison B. Hopelessness and eventual suicide: a 10 -year prospective study of patients hospitalized with suicidal ideation. Am J Psychiatry 1985;142:559-63. 
5. Beck AT, Brown G, Steer RA. Prediction of eventual suicide in psychiatric inpatients by clinical ratings of hopelessness. Journal of Consulting \& Clinical Psychology 1989;57:309-10.

6. Barraclough BM, Pallis DJ. Depression followed by suicide: a comparison of depressed suicides with living depressives. Psychol Med 1975;5:55-61.

7. Diekstra RFW. The epidemiology of suicide and parasuicide. Acta Psychiatrica Scandinavica 1993;371:9-20.

8. Mann JJ. The neurobiology of suicide. Nature Medicine 1998;4:25-30.

9. Mann JJ, Oquendo M, Underwood MD, Arango V. The neurobiology of suicide risk: a review for the clinician. J Clin Psychiatry 60 Suppl 1999;2:7-11; discussion 18-20, 113-6.

10. Barraclough B, Bunch J, Nelson B, Sainsbury P. A hundred cases of suicide: clinical aspects. Br J Psychiatry 1974;125:355-73.

11. Harris EC, Barraclough B. Suicide as an outcome for mental disorders. A meta-analysis. Br J Psychiatry 1997;170:205-28.

12. Lesage AD, Boyer R, Grunberg F, Vanier C, Morissette R, MenardButeau C et al. Suicide and Mental Disorders: A Case-Control Study of Young Men. American Journal of Psychiatry 1994;151:1063-8.

13. Malone KM, Haas GL, Sweeney JA, Mann JJ. Major depression and the risk of attempted suicide. Journal of Affective Disorders 1995;34:173-85.

14. Pendse B, Westrin A, Engstrom G. Temperament traits in seasonal affective disorder, suicide attempters with non-seasonal major depression and healthy controls . J Affect Disord 1999;54:55-65.

15. Weissman M, Fox K, Klerman GL. Hostility and depression associated with suicide attempts. Am J Psychiatry 1973;130:450-5.

16. Stein D, Apter A, Ratzoni G, Har-Even D, Avidan G. Association between multiple suicide attempts and negative affects in adolescents. J Am Acad Child Adolesc Psychiatry 1998;37:488-94.

17. Corruble E, Damy C, Guelfi JD. Impulsivity: a relevant dimension in depression regarding suicide attempts? J Affect Disord 1999;53:211-5.

18. Arango V, Ernsberger P, Marzuk PM, Chen JS, Tierney H, Stanley M, Reis DJ, et al. Autoradiographic demonstration of increased serotonin 5-HT2 and beta-adrenergic receptor binding sites in the brain of suicide victims. Arch Gen Psychiatry 1990;47:1038-47.

19. Hrdina PD, Demeter E, Vu TB, Sotonyi P, Palkovits M. 5-HT uptake sites and 5-HT2 receptors in brain of antidepressant- free suicide victims/depressives: increase in 5-HT2 sites in cortex and amygdala. Brain Res 1993;614:37-44.

20. Kraemer GW, Schmidt DE, Ebert MH. The behavioral neurobiology of self-injurious behavior in Rhesus monkeys: Current concepts and relations to impulsive behavior in humans. Ann N Y Acad Sci 1997;836:12-38.

21. Higley JD, Linnoila M. Low central nervous system serotonergic activity is traitlike and correlates with impulsive behavior. A nonhuman primate model investigating genetic and environmental influences on neurotransmission. Ann N Y Acad Sci 1997;836:39-56.

22. Higley JD, Thompson WW, Champoux M, Goldman D, Hasert MF, Kraemer GW et al. Paternal and maternal genetic and environmental contributions to cerebrospinal fluid monoamine metabolites in rhesus monkeys (Macaca mulatta). Arch Gen Psychiatry 1993;50:615-23.

23. Brent DA, Bridge J, Johnson BA, Connolly J. Suicidal behavior runs in families. A controlled family study of adolescent suicide victims. Arch Gen Psychiatry 1996;53:1145-52.

24. Johnson BA, Brent DA, Bridge J, Connolly J. The familial aggregation of adolescent suicide attempts. Acta Psychiatr Scand 1998;97:18-24.
25. Murphy GE, Wetzel RD. Family history of suicidal behavior among suicide attempters. The Journal of Nervous and Mental Disease 1982;170:86-90

26. Tsuang M. Risk of suicide in the relatives of schizophrenics, manics, depressives, and controls. Journal of Clinical Psychiatry 1983;44:396-400.

27. Egeland JA, Sussex JN. Suicide and Family loading for affective disorders. Journal of the American Medical Association 1985;254:915-8.

28. Roy A, Segal NL, Centerwall BS, Robinette CD. Suicide in twins. Arch Gen Psychiatry 1991;48:29-32X.

29. Schulsinger F, Kety S, Rosenthal D, Wender P. A family study of suicide, Orlando 1979.

30. Statham DJ, Heath AC, Madden PA, Bucholz KK, Bierut L, Dinwiddie SH et al. Suicidal behaviour: an epidemiological and genetic study. Psychol Med 1998;28:839-55.

31. Roy A, Segal NL, Sarchiapone M. Attempted suicide among living co-twins of twin suicide victims. Am J Psychiatry 1995;152:1075-6.

32. Papadimitriou GN, Linkowski P, Delarbre C, Mendlewicz J. Suicide on the paternal and maternal sides of depressed patients with a lifetime history of attempted suicide. Acta Psychiatrica Scandinavica 1991;83:417-49X.

33. Holland T, Gosden C. A balanced chromosomal translocation partially co-segregating with psychotic illness in a family. Psychiatry Research 1990;32:1-8.

34. Nielsen DA, Goldman D, Virkkunen M, Tokola R, Rawlings R, Linnoila M. Suicidality and 5-hydroxyindoleacetic acid concentration associated with a tryptophan hydroxylase polymorphism. Arch Gen Psychiatry 1994;51:34-8X.

35. Mann JJ, Malone KM, Nielsen DA, Goldman D, Erdos J, Gelernter J. Possible association of a polymorphism of the tryptophan hydroxylase gene with suicidal behavior in depressed patients. Am J Psychiatry 1997;154:1451-3.

36. Nielsen DA, Virkkunen M, Lappalainen J, Eggert M, Brown GL, Long JC et al. A tryptophan hydroxylase gene marker for suicidality and alcoholism. Arch Gen Psychiatry 1998;55:593-602.

37. Tzenova J, Rouleau GA, Dewar K, Lesage A, Vanier C, Lipp O et al.TPH and completed suicide. Submitted

38. Kunugi H, Ishida S, Kato T, Sakai T, Tatsumi M, Hirose T et al. No evidence for an association of polymorphisms of the tryptophan hydroxylase gene with affective disorders or attempted suicide among Japanese patients. Am J Psychiatry 1999;156:774-6

39. Furlong RA, Ho L, Rubinsztein JS, Walsh C, Paykel ES, Rubinsztein DC. No association of the tryptophan hydroxylase gene with bipolar affective disorder, unipolar affective disorder, or suicidal behaviour in major affective disorder. Am J Med Genet 1998;81:245-7.

40. Manuck SB, Flory JD, Ferrell RE, Dent KM, Mann JJ, Muldoon MF. Aggression and anger-related traits associated with a polymorphism of the tryptophan hydroxylase gene. Biol Psychiatry 1999;45:603-14.

41. New AS, Gelernter J, Yovell Y, Trestman RL, Nielsen DA, Silverman $\mathrm{J}$ et al. Tryptophan hydroxylase genotype is associated with impulsive-aggression measures: a preliminary study. Am J Med Genet 1998;81:13-7.

42. Kelly TM, Mann JJ. Validity of DSM-III-R diagnosis by psychological autopsy: a comparison with clinician ante-mortem diagnosis. Acta Psychiatr Scand 1996;94:337-43.

43. Turecki G, Brière R, Dewar K, Antonetti T, Lesage A, Séguin M et al. Prediction of level of serotonin $2 \mathrm{~A}$ receptor binding by serotonin receptor $2 \mathrm{~A}$ genetic variation in postmortem brain samples from subjects who did or did not commit suicide. American Journal of Psychiatry 1999;156:1456-8

Correspondência: Gustavo Turecki

Department of Psychiatry - McGill University, Douglas Hospital, 6875, LaSalle Boulevard - Verdun, Qc H4H 1R3 Email: Canadagustavo@bagel.epi.mcgill.ca 